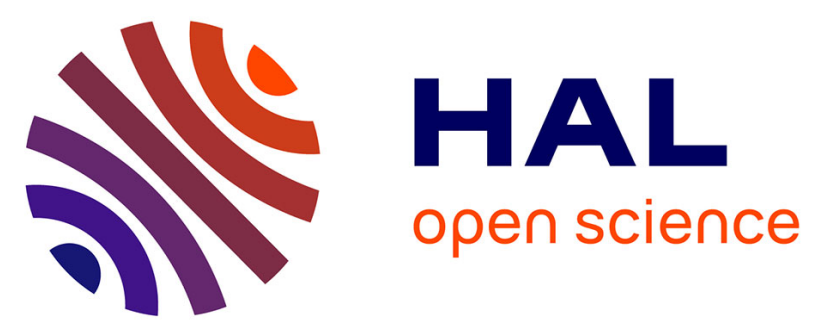

\title{
PEM fuel cell prognostics under variable load: a data-driven ensemble with new incremental learning
}

Kamran Javed, Rafael Gouriveau, Noureddine Zerhouni, Daniel Hissel

\section{To cite this version:}

Kamran Javed, Rafael Gouriveau, Noureddine Zerhouni, Daniel Hissel. PEM fuel cell prognostics under variable load: a data-driven ensemble with new incremental learning. International Conference on Control, Decision and Information Technologies, Apr 2016, Saint Julians, Malta. hal-02472570

\section{HAL Id: hal-02472570 \\ https://hal.science/hal-02472570}

Submitted on 10 Feb 2020

HAL is a multi-disciplinary open access archive for the deposit and dissemination of scientific research documents, whether they are published or not. The documents may come from teaching and research institutions in France or abroad, or from public or private research centers.
L'archive ouverte pluridisciplinaire HAL, est destinée au dépôt et à la diffusion de documents scientifiques de niveau recherche, publiés ou non, émanant des établissements d'enseignement et de recherche français ou étrangers, des laboratoires publics ou privés. 


\title{
PEM fuel cell prognostics under variable load: a data-driven ensemble with new incremental learning
}

\author{
Kamran Javed, Rafael Gouriveau, Noureddine Zerhouni, Daniel Hissel \\ FEMTO-ST Institute UBFC /CNRS /ENSMM /UTBM Besançon, France, \\ FCLAB (FR CNRS 3539) Belfort, France. \\ Email: firstname.lastname@femto-st.fr
}

\begin{abstract}
Proton Exchange Membrane Fuel cells (PEMFC) are one of the most promising fuel cell technologies, which qualify for variety of applications as power generation source. The Prognostics \& Health Management of fuel cell is an emerging field, which is paving the way for large scale industrial deployment of PEMFC technology. More precisely, prognostics of PEMFC become a major area of focus nowadays that enables predicting the behavior of PEMFC to produce actionable information to extend its life span. This paper contributes the first application on data-driven prognostics of PEMFC stack under variable load for combined heat and power generation ( $\mu$ CHP). In brief, an ensemble structure of Summation Wavelet-Extreme Learning Machine models is proposed with a new incremental learning scheme, to achieve long-term predictions on stack state of health (SOH) and to give confidence for better decisions. The proposed prognostics model is validated on data from PEMFC stack used for a $\mu$ CHP application under variable load profile for a complete year. A thorough comparison on SOH predictions results clearly shows the significance of proposed prognostics model, which can predict with few learning data for a long-term prognostics horizon around 650 hours with high accuracy and low uncertainty.
\end{abstract}

\section{INTRODUCTION}

Fuel cell (FC) is an energy system that offer clean and efficient way to generate electricity. The PEMFCs are considered the most versatile among available fuel cell technologies due to the advantages like: low operating temperature, high energy output and low pollutant emissions [1]. Hence, due to those advantages, PEMFCs qualify as a promising source for power generation for stationary, portable, and transportation applications, Fig. 1. However, FC aging is an unavoidable process, the optimization of its service and minimization of its life cycle costs/ risks require continuous monitoring of the degradation process and accurate prediction of its future behavior to enable timely decisions. In this context, Prognostics and Health Management (PHM) of FCs is an emerging field, which aims extending their life span, while reducing exploitation and maintenance costs. Therefore, paving the way for large scale industrial deployment of PEMFC technology. More precisely, PEMFC prognostics becomes a major area of focus nowadays, as a key task in PHM cycle with future capabilities.

In brief, prognostics uses condition monitoring data (CM) from PEMFC stack (i.e., assembly of elementary cells) to identify degradation at early stages, to predict long-term behavior and to estimate its remaining useful life (RUL). This will enable, short term, medium term or long term decisions to prolong the life span of the fuel cell/ stack. Such decisions can be related to control, maintenance or changing mission profiles.

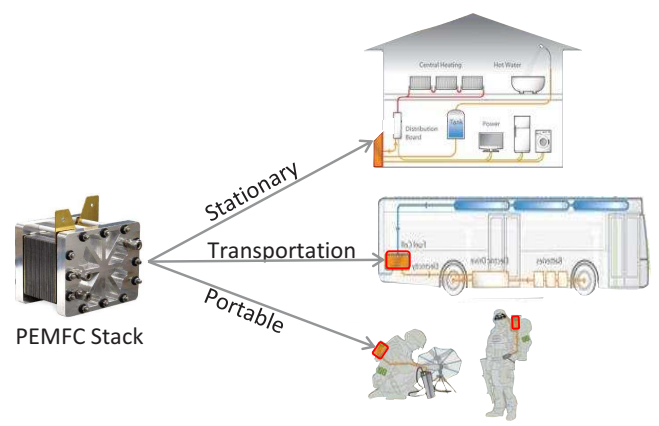

Fig. 1. PEM Fuel Cell applications

According to author's knowledge, few works have been published on prognostics of PEMFC on stack level. Those works can be classified into two main categories, 1) prognostics under constant load current, and 2) prognostics under variable loads current. The methods that belong to the first category are data-driven prognostics approaches, namely: SummationWavelet Extreme Learning Machine [1], Echo state network (ESN) [2], Adaptive Neuro-Fuzzy Inference System (ANFIS) [3] and Particle Filtering framework [4]. In the second category only one method has been published using a hybrid prognostics approach on PEMFC for a stationary application for combined heat and power for small buildings ( $\mu \mathrm{CHP})$ Fig. 1, [5]. Unfortunately, no work has been done so far on datadriven prognostics of PEMFC under variable load. This is due to quantity and quality CM data from PEMFC stack, which prevents building data-driven model that are robust enough to manage the uncertainty of data and modeling phase. Also data-driven approaches under constant loads are mainly based on the assumption that stack aging is irreversible degradation, which limits applicability of those methods for variable load. To address those issues, this paper presents first application on data-driven prognostics of PEMFC under variable load current for $\mu$ CHP. In brief, a data-driven ensemble of Summation Wavelet-Extreme Learning Machine models is proposed with new incremental learning scheme to improve the adaptability of the model (online), to achieve accurate long-term predictions of PEMFC state of health (SOH) and to give confidence to predictions. The main contributions of this development are:

- $\quad$ ensemble structure to manage uncertainty;

- $\quad$ incremental learning to improve adaptability;

- $\quad$ application on PEMFC stack under variable load. 
The remaining paper is organized as follows. Section II elaborates issues related to CM data under constant vs. variable loads and modeling. It also highlights the importance of incremental learning and uncertainty related tasks for prognostics. Section III presents the proposed data-driven connectionist ensemble with incremental learning scheme. Section IV validates our proposition on PEMFC stack under variable loads for $\mu \mathrm{CHP}$ and compares with hybrid approach [5]. Section V concludes this work and gives future perspectives.

\section{CM DATA, MODELING, UNCERTAINTY: CHALLENGES}

The CM data are fundamental to implement a right model for health assessment, prognostics and to estimate its parameters. Such data are collected at regular intervals through a procedure of monitoring carefully selected parameters of health condition/ state of the equipment. CM data that can be continuously measured (or acquired), controlled and economically possible from PEMFC stack are: aging time $(t)$, stack voltage $(V)$, stack power $(P)$, current $(\mathrm{A})$, air/ $H_{2} /$ cooling water temperatures and air compressor speed. Among those data, stack voltage/ power are considered as useful indicators to assess the condition of the stack and to predict its degrading behavior. However, such data are noisy and non-stationary even under constant current, whereas variable load current poses an additional challenge for data-driven approaches. Fig. 2 depicts this issue with a clear example by comparing power drop signals from two stacks under constant and variable loads. Both stacks show a totally different aging behavior.

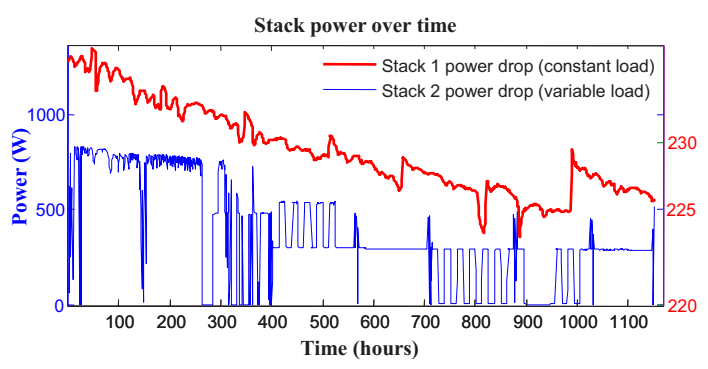

Fig. 2. Comparing PEMFC stacks power drop signals

The data-driven methods for prognostics of PEMFC under constant loads ([1], [2], [3], [4]), are mainly based on the assumption, that stack aging process is an irreversible degradation. Therefore, prognostics with those methods is performed by projecting the stack voltage/ power trend (e.g. see [1]). Also they did not use current as an input to the prognostics model, knowing that it will remain constant throughout the life of PEMFC stack. However, such assumptions limit the applicability of data-driven methods for PEMFC prognostics under varying load. Nevertheless, using current as an input to the prognostics model can enable learning the relation between inputs and targets [6]. The learned model is further used to assess the current condition of the PEMFC stack and to predict its aging behavior with changing current profile.

However, due to limited learning data, measurement noise, model inaccuracy, etc., a single model is incapable to encounter uncertainty related to input data and to accurately predict stack aging over long prediction horizon. As a result, the predicted response of the model is different from true response and thus the prognostics is uncertain. The uncertainty in the case of health assessment and prognostics is called as modeling uncertainty, which can be reduced by improved methods [7]. Finally, whatever the type of uncertainty, it should be quantified and propagated to give confidence to predictions, which facilitates offline/ online decisions to prolong the life span of the stack (i.e., by control, maintenance or changing mission profiles). According to above discussions and challenges, following points are vital for efficient prognostics of PEMFC.

1) Data are the key source of information that must be used intelligently to manage uncertainty.

2) Multidimensional data is essential for prognostics.

3) Operating conditions that correlate degradation, should be used as inputs.

4) Prognostics model should be capable of updating its parameters in real-time when new data are available.

5) Uncertainty of the prognostics must be quantified and propagated to give confidence for decisions.

\section{PROPOSED DATA-DRIVEN PROGNOSTICS APPROACH}

FCs are highly multiphysics (due to electrical, mechanical, thermal engineering, etc.,), multiscale systems (from $\mu \mathrm{m}$ to $m$ ), and it is not easy to access their internal parameters or to fully understand their aging process. Therefore, building an accurate physics based prognostics models can be very hard. Nevertheless, with the advance of modern sensor, data storage and processing technologies, the data-driven prognostics models are becoming popular [8], which can also serve as powerful tools to ensure safety and availability of degrading PEMFC. They learn FC/ stack behavior directly from CM data without detailed physical understanding about the aging process, and that knowledge is used to predict degradation.

According to discussions on CM data and modeling in previous section, the framework of PEMFC health assessment and prognostics can be easily understood by Fig. 3. Therefore, discussions on data-acquisition and data-processing steps are left aside. As for prognostics modeling, the data-driven model learns directly stack aging behavior from selected health indicators, which are stack aging time, current and stack power drop in our case. The learned model is used at time $(t)$ to assess current SOH of the PEMFC stack, and to predict the evolution $\mathrm{SOH}$ from $t \rightarrow t+h$, where $h$ is the prediction horizon. This enables timely decisions to prolong its life span by performing maintenance or changing mission profiles.

On the basis of our previous development for prognostics of PEMFC [1], and considering the requirements highlighted in sec. II: for the prognostics modeling step an adaptive ensemble of Summation Wavelet-Extreme Learning Machine algorithm (SW-ELM) is proposed with new incremental learning scheme to predict stack degradation under given variable load profile.

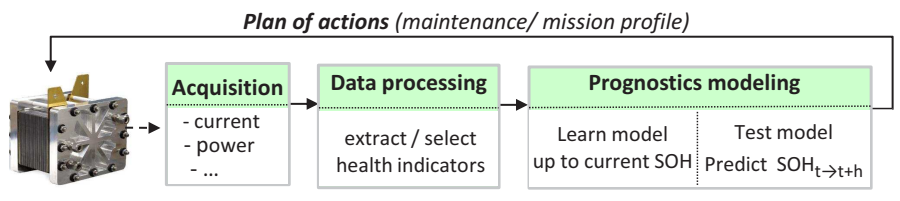

Fig. 3. From CM data to long-term prediction of PEMFC stack $\mathrm{SOH}$ 


\section{A. Overview of basic SW-ELM \& mathematical perspective}

The SW-ELM combines neural network and wavelet theory for estimation/ predictions problems [9]. Basically, SW-ELM is one-pass batch learning algorithm for single layer feed forward network (SLFN), Fig. 4. It has an advantage of improved parameter initialization to reduce impact of random weights and bias of input-hidden layer, and an improved structure with two activation functions in each hidden node to encounter nonlinear inputs. Also it works on actual scales of the data.

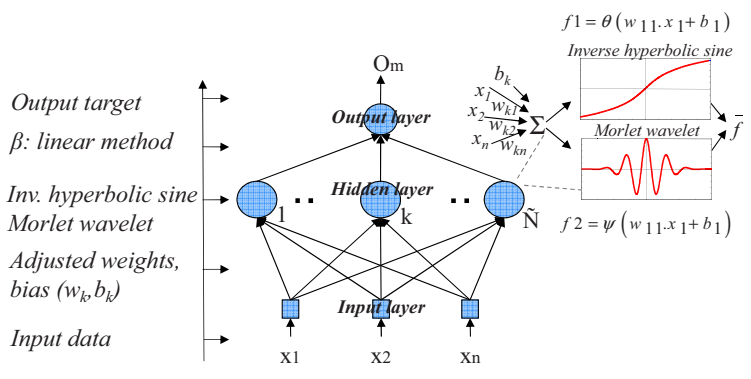

Fig. 4. Machine learning view of SW-ELM

Let note $n$ and $m$ the numbers of inputs and outputs, $N$ the number of learning data samples $\left(x_{i}, t_{i}\right)$, where $i \in[1 \ldots N]$, $x_{i}=\left[x_{i 1}, x_{i 2}, \ldots, x_{i n}\right]^{T} \in \Re^{n}$ and $t_{i}=\left[t_{i 1}, t_{i 2}, \ldots, t_{i m}\right]^{T} \in$ $\Re^{m}$, and $\tilde{N}$ the number of hidden nodes, each having activation functions $\left(f_{1} \& f_{2}\right)$. To minimize the difference between output $o_{j}$ and target $t_{j}$, there exist $\beta_{k}, w_{k}$ and $b_{k}$ such that:

$$
\sum_{k=1}^{\tilde{N}} \beta_{k} \bar{f}\left[(\theta, \psi)\left(w_{k} . x_{j}+b_{k}\right)\right]=t_{j}, j=1,2, \ldots, N
$$

where $\bar{f}$ is the average output from two different activation functions $\theta$ and $\psi . w_{k}=\left[w_{k 1}, w_{k 2}, \ldots, w_{k n}\right]^{T} \in \Re^{n}$ is an input weight vector connecting the $k^{\text {th }}$ hidden to input layer neurons, $\left(w_{k} \cdot x_{j}\right)$ is the inner product of weights and inputs, and $b_{k} \in \Re$ is the bias of $k^{t h}$ hidden neuron. Also, $\beta_{k}=\left[\beta_{k 1}, \beta_{k 2}, \ldots, \beta_{k m}\right]^{T} \in \Re^{m}$ is the weight vector to connect $k^{t h}$ hidden neuron to output neuron. In matrix form Eq. 1 can be written as $H_{\text {avg }} \beta=T$, where $T$ is target matrix and $H_{\text {avg }}$ is hidden layer output matrix expressed as:

$$
\begin{gathered}
H_{\text {avg }}\left(w_{1}, \ldots, w_{\tilde{N}}, x_{1}, \ldots, x_{\tilde{N}}, b_{1}, \ldots, b_{\tilde{N}}\right)= \\
\bar{f}(\theta, \psi)\left[\begin{array}{ccc}
\left(w_{1} \cdot x_{1}+b_{1}\right) & \ldots & \left(w_{\tilde{N}} \cdot x_{1}+b_{\tilde{N}}\right) \\
\vdots & \ldots & \vdots \\
\left(w_{1} \cdot x_{N}+b_{1}\right) & \ldots & \left(w_{\tilde{N}} \cdot x_{N}+b_{\tilde{N}}\right)
\end{array}\right]_{N \times \tilde{N}} \\
\beta=\left[\beta_{1}^{T} \cdots \beta_{\tilde{N}}^{T}\right]_{\tilde{N} \times m} \text { and } T=\left[t_{1}^{T} \cdots t_{N}^{T}\right]_{N \times m}
\end{gathered}
$$

Finally, the least square solution of the linear system $H_{\text {avg }} \beta=$ $T$, with minimum norm of output weights $\beta$ is:

$$
\hat{\beta}=H_{\text {avg }}^{\dagger} T=\left(H_{\text {avg }}^{T} H_{\text {avg }}\right)^{-1} H_{\text {avg }}^{T} T
$$

where $H_{a v g}^{\dagger}$ shows the Moore-Penrose generalized inverse for the hidden layer output matrix $H_{\text {avg }}$ [10]. The SW-ELM algorithm can be synthesized as follows (see details in [9]).

\begin{tabular}{l}
\hline Algorithm 1 Brief learning scheme of SW-ELM \\
\hline Assume: $n$ inputs, $m$ outputs, $\tilde{N}$ hidden nodes \\
Require: $N$ learning samples, Arcsinh \& Morlet functions \\
1: Initialize wavelet parameters (i.e., dilatation $\&$ translation). \\
2: Initialize randomly hidden nodes param. $\left(w_{k}, b_{k}\right) \&$ adjust. \\
3: Obtain hidden layer output matrix $H_{\text {avg }}$ using Eq. 2. \\
4: Find the output weight matrix $\hat{\beta}$ in Eq. 4.
\end{tabular}

\section{B. Building the SW-ELM ensemble}

Predicting behavior of aging PEMFC stack is a complicated task, since there are various sources of uncertainty that impact predictions and the error accumulates with increasing prediction horizon. Considering the data-driven framework for prognostics of PEMFC in Fig. 3, at each step the uncertainties get compounded, that can grow beyond control in the prediction phase if not managed properly [11]. Therefore, considering uncertainties either due to data or either due to modeling phase (sec. II), a single model is not robust to reduce their impact, which could lead to wrong decisions. In comparison, performing prognostics with an ensemble of models would be less likely to be in error than an individual model and appears to be meaningful [1]. The ensemble approach enables reducing the uncertainty and gives confidence to the predictions to facilitate decisions. A narrow confidence indicates better performances of prognostics in terms of precision and accuracy over wide confidence which indicates large uncertainty.

The proposed ensemble structure is achieved by combining small groups of ensembles using SW-ELM. Each individual SW-ELM model has the same network complexity 5 input55 hidden-1 output neuron, but different learning parameters (algo. 1), see Fig. 5. The parameter constant $C$ to adjust $w_{k}$ and $b_{k}$ of each SW-ELM is set to $C=0.01$ (see [9]). The inputs of each SW-ELM are stack aging time $(t)$, current (I), 3 regressors of stack power $\left(P_{t-2}, P_{t-1}, P_{t}\right)$. The number of SW-ELM models in each group is set to 10 , and the number of groups is set to 20 . The predicted output $\left(\hat{P}_{t+1}\right)$ from the ensemble structure is the median value from medians computed from each group of SW-ELM models. This allows narrowing confidence of predicted values. To improve the adaptability of ensemble structure for better predictions and to manage uncertainty for better decisions, the incremental learning scheme with artificial data is presented as follows.

\section{New incremental learning scheme}

To perform long-term prognostics, a common practice is to project the current condition of equipment up to failure limit, assuming that predictions follow the degrading trend [1]. This task is usually achieved by an iterative approach, that is performed by a single model which is tuned to perform onestep ahead prediction $\hat{x}_{t+1}$. The predicted value is used as the regressors of the model to predict the following ones and the process is repeated until the prediction of $\hat{x}_{t+h}$ (see [12]). This process is repeated each time to evolve model parameters when new measurements are available, till the end of life of the equipment [1]. The whole procedure can be considered as incremental learning, because the prognostics models are re-trained and their parameters are updated according to new 


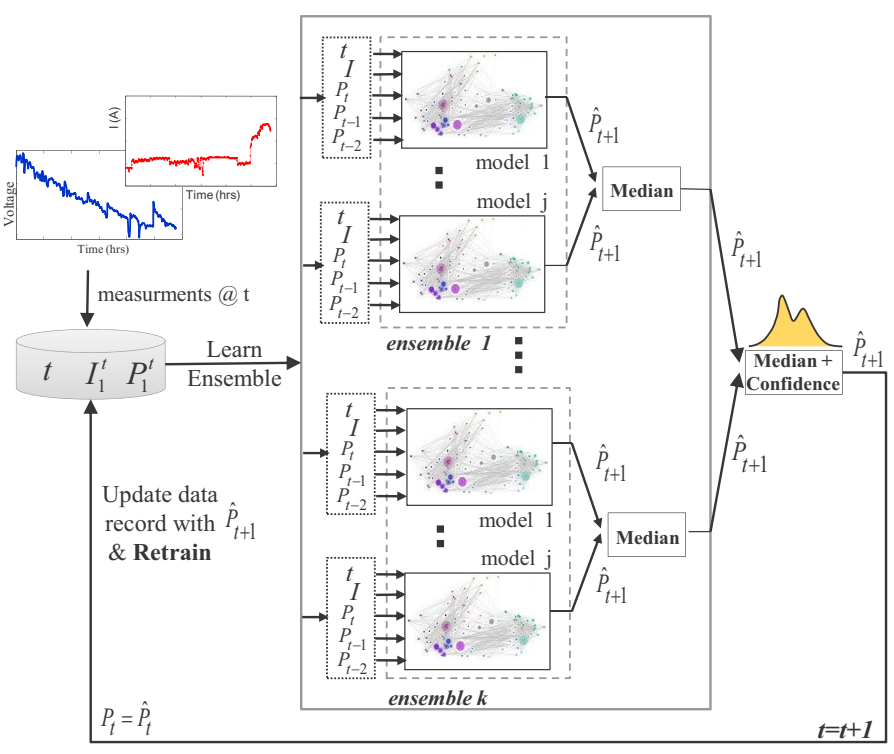

Fig. 5. Ensemble structure \& learning strategy

data new [13]. The iterative approach can be useful for projection based prognostics methods under constant operating conditions, however, under variable load the assumption of degrading trends does not apply. Also with iterative approach the error will accumulate at each time step, which can result large uncertainty of prognostics. Therefore, main issues are: 1) how to do incremental learning without new data?, 2) how to manage uncertainty of long-term predictions?. To address those issues, the proposed approach for incremental learning uses the artificial data (or re-simulated data) from one-step ahead prediction model to update data record and the prediction model is retrained with those data Fig. 5. In order to elaborate the learning procedure of ensemble structure, consider $\mathrm{CM}$ data record from PEMFC stack with measurements from three health indicators up to time $t$, i.e., stack aging time $t$, current $I$ and 3 regressors from stack power $P$. Each SW-ELM model is trained with those data to perform one-step ahead prediction of power $\hat{P}_{t+1}$. The median prediction value $\hat{P}_{t+1}$ for ensemble structure is stored sequentially in the data record against the set of inputs at time $t$, which is considered as the artificial data from prediction to increase the learning frame. The ensemble structure is retrained with new sample (of artificial data) to update parameters, prior to the next prediction step. This procedure is repeated until the end of given current profile to predict the behavior of PEMFC stack SOH with changing current loads and to enable timely decisions. The proposition allows performing incremental learning without actual measurements and using artificial data from predictions, thus improving the adaptability of prognostics model and managing its uncertainty of long-term predictions.

When new measurements from the stack are available, the complete procedure of proposed incremental learning scheme is repeated. Note that, due to rapid learning ability of SWELM algorithm, the proposed incremental learning can be computationally efficient. However, the classical algorithms for SLFN, like backpropagation algorithm can be computationally expensive. Moreover, the computational time can increase with the complexity of ensemble structure.

\section{EXPERIMENT AND RESULTS DISCUSSION}

\section{A. PEMFC stack $C M$ data under $\mu$ CHP profile}

The proposed data-driven ensemble with incremental learning scheme is applied to prognostics of PEMFC stack of $1 \mathrm{~kW}$ for a $\mu \mathrm{CHP}$ application. PEMFC stack used for the experiment was composed of 8 cells, with an active area of $220 \mathrm{~cm}^{2}$. The mission profile to operate the stack for $\mu \mathrm{CHP}$ under variable load current for a complete year (Fig. 6a), was based on the on-field measurements from a demonstration project for which Electricite de France (eDF) was partner [14]. The CM data were collected from a $10 \mathrm{~kW}$ test bench at FCLAB Research Federation (FR CNRS 3539), Fig. 6b. The operating variables concerning, fluids (1), electrical (2), and thermal flows (3) were acquired from PEMFC (4) at a frequency of $1 \mathrm{~Hz}$. To recall, the data from PEMFC stack continuously measured, controlled and economically possible are: aging time $(t)$, stack power $(P)$ Fig. 2, current (A) Fig. 7a, stack voltage $(V)$ Fig. 7b, air/ $\mathrm{H}_{2} /$ cooling water temperatures and air compressor speed.
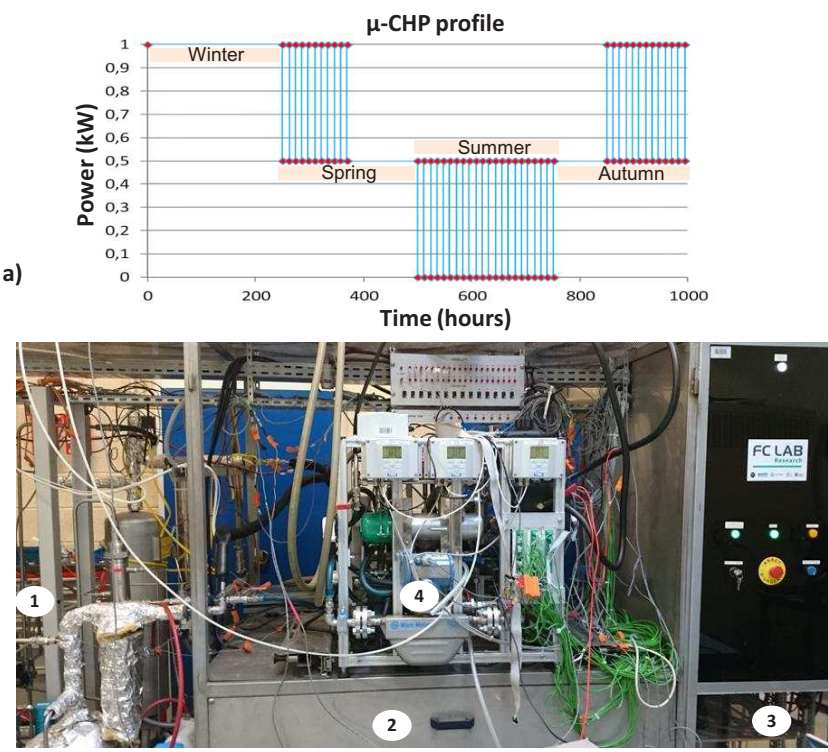

b)

Fig. 6. $\mu \mathrm{CHP}$ profile a) and $10 \mathrm{~kW}$ test bench $\mathrm{b}$ )
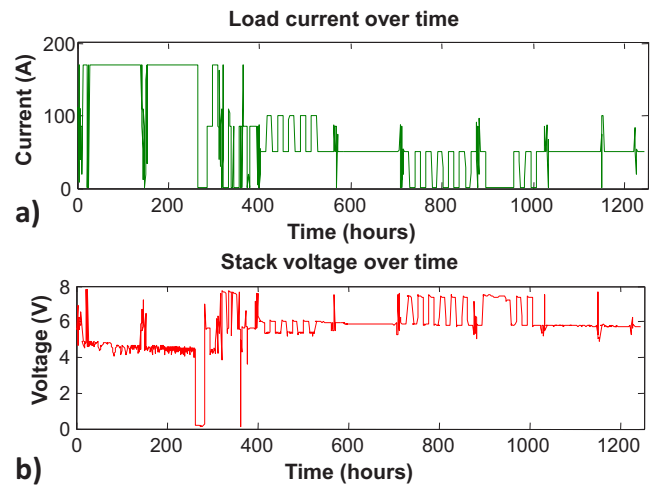

Fig. 7. Current and voltage measurements from PEMFC stack 
Without incremental learning
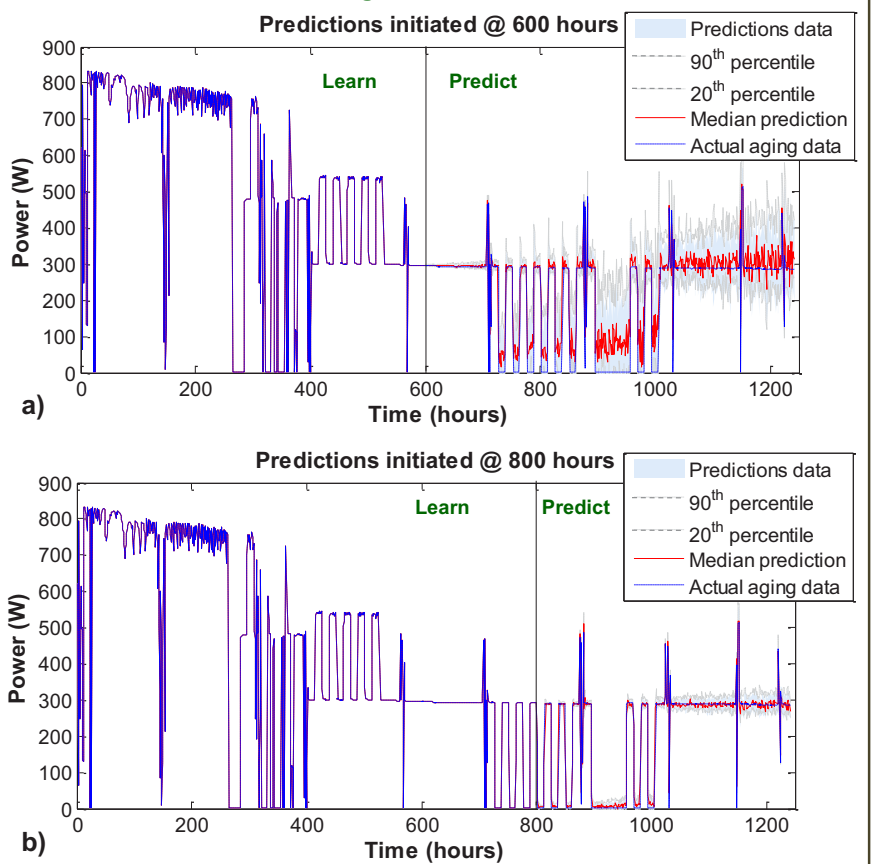

With incremental learning (using artificial data)
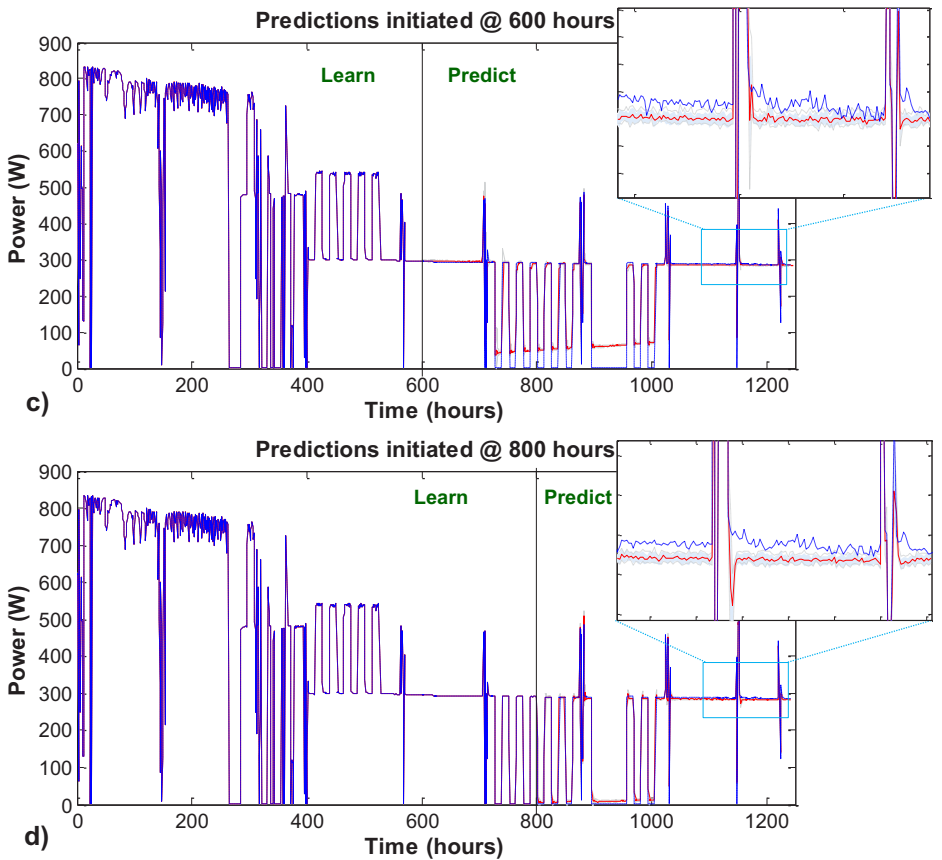

Fig. 8. Long-term prediction results of PEMFC stack SOH, a), b) without incremental learning \& c),d) with incremental learning

\section{B. SOH prediction results}

To validate our proposition, during the computer simulations prognostics is initiated around half-life of the PEMFC stack. As mentioned in sec. III, it is assumed that the future load demand of $\mu \mathrm{CHP}$ application is known for a complete year (i.e., for four seasons). Therefore, the current is used as an input to the prognostics model to adapt variation of the stack power over increasing prediction horizon. Like a real situation, prognostics is performed at two different time steps with different lengths of prediction horizons: 1) prognostics initiated at 600 hours, where the prediction horizon is 643 hours, 2) prognostics initiated at 800 hours, where the prediction horizon is 443 hours. The results are discussed and a comparison is given to show improvements. Most importantly, the proposed approach is compared with the recent works on hybrid prognostics of PEMFC under variable loads.

1) Without incremental learning: The long-term prediction results on SOH of PEMFC with SW-ELM ensemble (Fig. 5), without incremental learning are shown in Fig. 8a,b. Consider the first case, where predictions are initiated after learning measurements up to 600 hours. The median predictions from the ensemble can be seen with a red line. The large uncertainty of predictions over horizon of 643 hours is shown with a wide confidence around predictions, which is due to error accumulation at each step with iterative approach (sec. III). The median predictions from the ensemble are poor and they do not track properly behavior of stack (i.e. blue dotted line). For the second case Fig. 8b, predictions are initiated after learning measurements up to 800 hours. The median predictions from the ensemble are quite good and they track properly the actual aging behavior of the stack. Also, the uncertainty of predictions over the horizon of 443 hours is reduced as compared to the previous case. This indicates the requirement of large frame of learning data for the ensemble model.

2) With incremental learning: The long-term prediction results on SOH of PEMFC with data-driven ensemble (Fig. 5), with incremental learning are shown in Fig. 8c,d for which same legend apply as Fig. 8a,b. For clarity of results a zoom in view of predictions is given in spite of legend.

Like previous results, consider the first case, where predictions are initiated at 600 hours. Even with the few learning data, the median predictions from the ensemble follow the stack aging behavior quite well as compared to results in Fig. 8a. Moreover, the confidence around predictions is very compact over the entire horizon of 643 hours, as shown in zoom in view, which indicates the robustness of the prognostics model to encounter uncertainty. However, the predictions from 800 hours up to 1000 hours on the power drop signal are not very accurate, but still better than results in Fig. 8a.

For the second case Fig. 8d, predictions are initiated at 800 hours. The median predictions from the ensemble are quite accurate as they closely tracks actual aging behavior of the stack, which is also shown in the zoom in view. The uncertainty bounds of predictions are very narrow as well, over the entire horizon of 443 hours, which indicates higher precision and also the reliability of prognostics model.

3) Comparative analysis: The $\mathrm{SOH}$ predictions results given in Fig. 8 are further evaluated using coefficient of determination metric $(R 2)$, for both cases, i.e., without and with incremental learning. Basically, $R 2$ determines, how accurate the future outcomes are to be predicted by the learned model. The comparison of $R 2$ results is given in Fig. 9, for prognostics at 600,800 and 1000 hours. The comparison show that, the proposed incremental learning scheme can give more accurate prognostics results, when few learning data are available (i.e., up to 600 hours). Following that, the $R 2$ accuracies with both 
cases (without and with incremental learning) are same for prognostics at 800 hours and 1000 hours. However, it should be noted that, at those time steps without incremental learning, the predictions are less precise as compared to proposed incremental learning scheme, which is already mentioned in sec. IV-B.

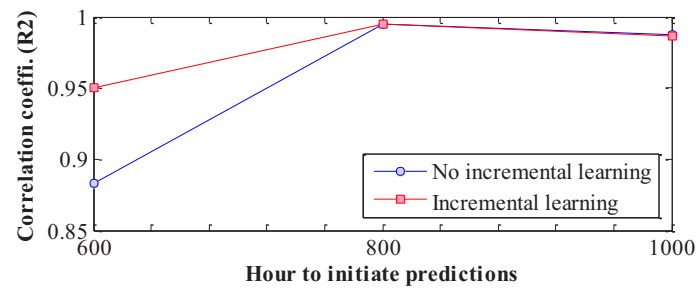

Fig. 9. Comparing prediction accuracy using $R 2$.

The results of prognostics accuracy with the proposed datadriven ensemble with new incremental learning scheme are further compared with the hybrid approach on prognostics of PEMFC under variable load [5].

The $R 2$ results from the hybrid prognostics model are given shown in Fig. 10. For prognostics at 600 hours and 800 hours, with proposed approach the accuracy results are $R 2=0.949$ and $R 2=0.995$ respectively, whereas with hybrid prognostics at 600 hours and 800 hours, accuracy results are $R 2=0.68$ and $R 2=0.73$. These results clearly show the significance of the proposed data-driven ensemble with incremental learning.

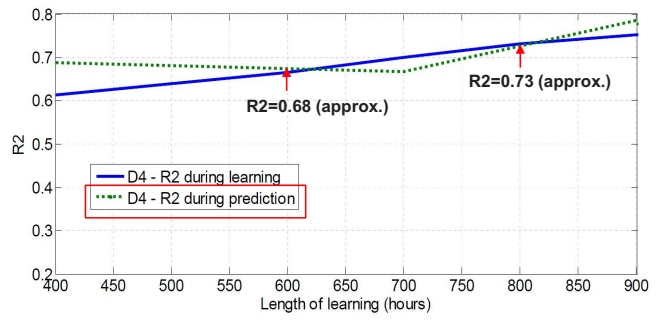

Fig. 10. Evaluating prediction accuracy using $R 2$ [5]

\section{CONCLUSION}

In this paper a data-driven approach is proposed for prognostics of PEMFC stack for combined heat and power generation $(\mu \mathrm{CHP})$. The proposed prognostics model is based on Summation Wavelet-Extreme Learning Machine (SW-ELM) ensemble with a new incremental learning scheme for an online application. The SW-ELM ensemble is applied to monitoring data from PEMFC stack under known variable loads, which is the first application from data-drive category of prognostics approaches. Long-term prognostics on state of health $(\mathrm{SOH})$ of the PEMFC is performed with few learning data. The $\mathrm{SOH}$ prediction results with proposed data-driven approach, and its comparison with hybrid prognostics, clearly show the significance of SW-ELM ensemble model which predicts with higher accuracy and lower uncertainty over longterm prognostics horizon. The future developments of this work concern the application of proposed data-driven ensemble on prognostics of PEMFC for transportation application.

\section{ACKNOWLEDGMENT}

This work is supported by the ANR project PROPICE (ANR-12-PRGE-0001) and by the Labex ACTION project (contract "ANR-11-LABX-01-01") both funded by the French National Research Agency.

\section{REFERENCES}

[1] K. Javed, R. Gouriveau, N. Zerhouni, and D. Hissel, "Improving accuracy of long-term prognostics of pemfc stack to estimate remaining useful life," in Industrial Technology (ICIT), 2015 IEEE International Conference on, 2015, pp. 1047-1052.

[2] S. Morando, S. Jemei, R. Gouriveau, N. Zerhouni, and D. Hissel, "Fuel cells prognostics using echo state network," in Industrial Electronics Society, IECON 2013-39th Annual Conference of the IEEE, 2013, pp. 1632-1637.

[3] R. Silva, R. Gouriveau, S. Jemeï, D. Hissel, L. Boulon, K. Agbossou, and N. Y. Steiner, "Proton exchange membrane fuel cell degradation prediction based on adaptive neuro-fuzzy inference systems," International Journal of Hydrogen Energy, vol. 39, no. 21, pp. 11 128-11 144, 2014.

[4] M. Jouin, R. Gouriveau, D. Hissel, M.-C. Péra, and N. Zerhouni, "Prognostics of pem fuel cell in a particle filtering framework," International Journal of Hydrogen Energy, vol. 39, no. 1, pp. 481-494, 2014.

[5] M. Jouin, R. Gouriveau, D. Hissel, M. C. Pra, and N. Zerhouni, "Prognostics of prm fuel cells under a combined heat and power profile," IFAC-Papers OnLine, vol. 48, no. 3, pp. 26 - 31, 2015, 15th IFAC Symposium on Information Control Problems in Manufacturing INCOM 2015

[6] M. Gašperin, P. Boškoski, and D. Juricic, "Model-based prognostics under non-stationary operating conditions," in Annual Conference of the Prognostics and Health Management Society, 2011, pp. 831-853.

[7] J. R. Celaya, A. Saxena, and K. Goebel, "Uncertainty representation and interpretation in model-based prognostics algorithms based on kalman filter estimation," in Annual Conference of the Prognostics and Health Management Society, 2012.

[8] C. Hu, B. D. Youn, P. Wang, and J. T. Yoon, "Ensemble of data-driven prognostic algorithms for robust prediction of remaining useful life," Reliability Engineering \& System Safety, vol. 103, pp. 120-135, 2012.

[9] K. Javed, R. Gouriveau, and N. Zerhouni, "SW-ELM: A summation wavelet extreme learning machine algorithm with a priori parameter initialization," Neurocomputing, vol. 123, pp. 299-307, 2014.

[10] D. Serre, What Are Matrices. Springer, 2010.

[11] A. Saxena, J. R. Celaya, I. Roychoudhury, S. Saha, B. Saha, and K. Goebel, "Designing data-driven battery prognostic approaches for variable loading profiles: Some lessons learned," in European Conference of Prognostics and Health Management Society, 2012, pp. 1-11.

[12] R. Gouriveau and N. Zerhouni, "Connexionist-systems-based long term prediction approaches for prognostics," IEEE Trans. Rel., vol. 61, no. 4, pp. 909-920, 2012.

[13] R. Polikar, L. Udpa, S. S. Udpa, and V. Honavar, "Learn++: an incremental learning algorithm for multilayer perceptron networks," in Acoustics, Speech, and Signal Processing, 2000. ICASSP'00. Proceedings. 2000 IEEE International Conference on, vol. 6, 2000, pp. 34143417.

[14] P. Mocoteguy and B. Al-Nasrawi, "Field testing of vaillant euro 2 pem fuel cell heating appliance for $\mu-c h p$ in residential application," in Workshop on degradation issues in Fuel Cells, Hersonissos, Crete, 2007. 